\title{
Application of high frequency filtration to remove cloudiness in Earth remote sensing images
}

\author{
Valeriy Tutatchikov ${ }^{1 *}$, Mikhail Noskov ${ }^{1}$ \\ ${ }^{1}$ Siberian Federal University, School of Space and Information Technology, 660074, Krasnoyarsk, \\ Russian Federation
}

\begin{abstract}
At present, methods of digital processing of Earth remote sensing images are widely used to improve the image quality. For example, many images are discarded due to high clouds in the images, which obscure objects of interest. In this paper, the possibility of using highfrequency global filters to reduce cloudiness in the image is considered, and the results of image enhancement are shown.
\end{abstract}

\section{Introduction}

At present, methods of digital processing of Earth remote sensing (ERS) images are widely used to improve the image quality. But many original images contain distortions, for example, high clouds, which partially or completely hide objects of interest. The possibility of preliminary ranking or selection of images with respect to the prevalence and location of clouds in the image should significantly reduce the search time for suitable images for solving applied problems [1].

Another common way to improve such images is preliminary processing of remote sensing data: radiometric, atmospheric or geometric correction [2]. It is also possible to use classical methods of spatial filtering of digital image processing [3]. In this paper, we will consider the possibility of applying global high-frequency filtering methods to eliminate cloudiness of remote sensing images.

\section{Method description}

Consider, as the initial data, a snapshot of remote sensing of the Earth of the vicinity of the Taseevsky district of the Krasnoyarsk Territory (Figure 1). As a distortion of the cloudiness of the image, the smoke in this area is presented, and the central part of the image contains a high degree of distortion, and the lateral left one is already less [4].

\footnotetext{
*Corresponding author: vtutatchikov@mail.ru
} 


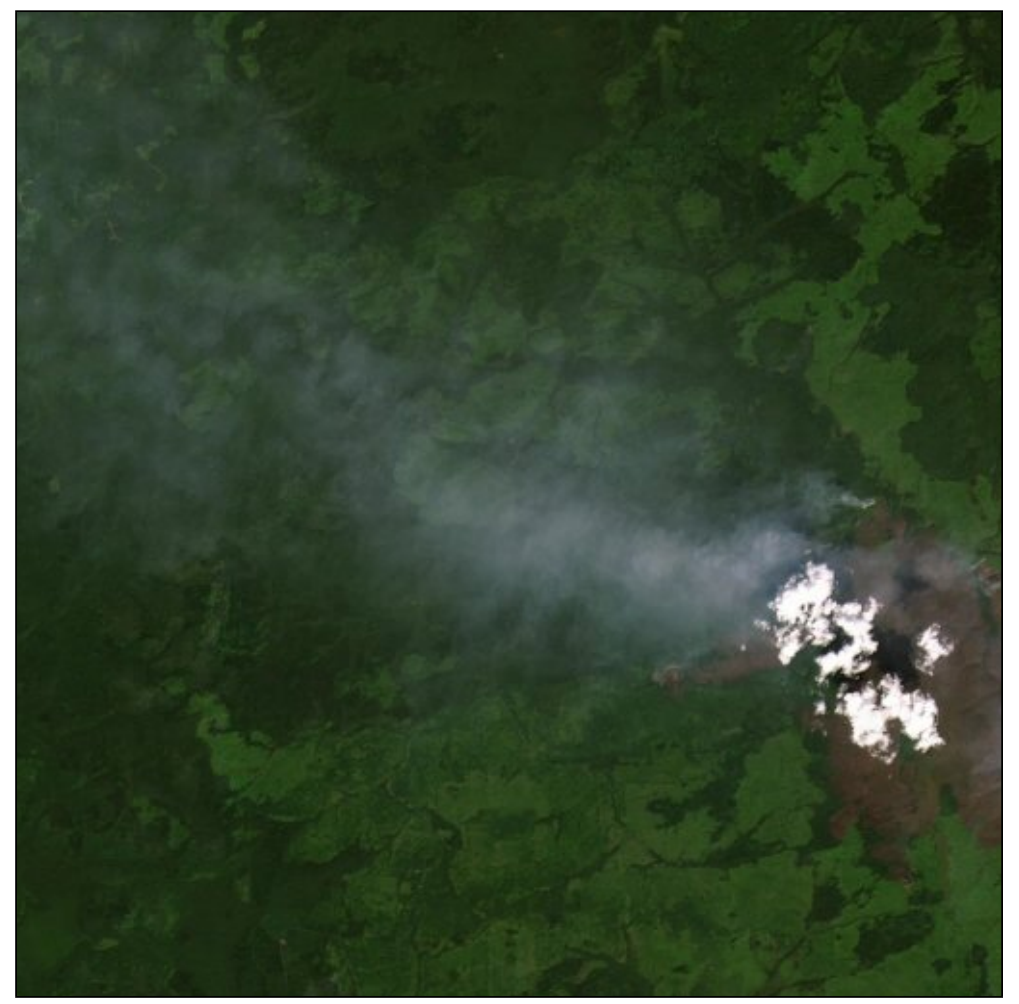

Fig. 1. Surface snapshot with smoke

For the convenience of calculations, let us set the size of the investigated area as a power of two: $512 * 512$ pixels (the use of the Fourier transform of two-dimensional signals with a number of samples other than a power of two is described in [5-6]). This image can be represented as a function $f(x, y)$ of the brightness of a pixel with coordinates $x$ and $y$, taking integer values 0-255 for each red, green, blue channel in the RGB color model.

In [3], it is proposed to multiply all the image elements $f(x, y)$ by $(-1)^{x+y}$, and then calculate the discrete Fourier transform of this function. In this case, we get a centered Fourier transform $F(u, v)$ in the frequency domain, in which high frequencies (responsible for image contours and sharp transitions) will be in the center of the spectrum, and low frequencies (responsible for image smoothing and color saturation) will be around the edges.

We apply a global high-pass filter to the obtained function $F(u, v)$, for example, a Gaussian high-pass filter (HPF):

$$
H(u, v)=1-\exp \left(\frac{-D^{2}(u, v)}{2 \cdot D_{0}^{2}}\right)
$$

where $D(u, v)$ is the distance from the center of the Fourier transform to the point with coordinates $(u, v), D_{0}$ is the cutoff frequency. The Gaussian HPF graph is shown in Figure 2. 


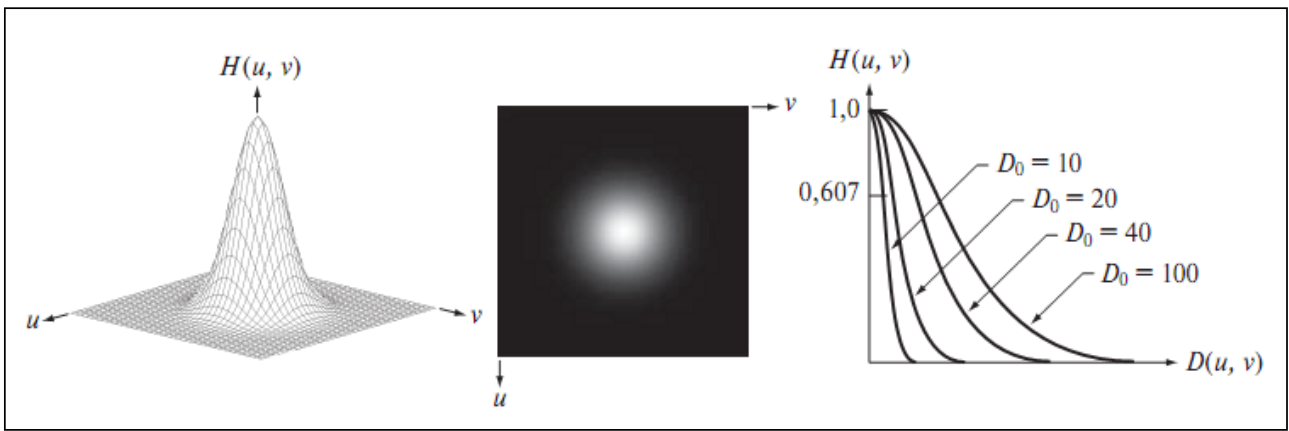

Fig. 2. Graph of a Gaussian LPF function on the left, a grayscale image of the filter in the center, radial filter profiles for different values of $D_{0}$ on the right

As a result of filtering, the high frequencies will remain unchanged, and the low frequencies outside the radius $D_{0}$ will be zeroed. After that, we calculate the twodimensional inverse Fourier transform, multiply all samples of the resulting function by $(-1)^{x+y}$ to cancel centering, and get the processed image $f_{l}(x, y)$.

\section{Obtained results}

The result of filtering is shown in Figure 3. The image has sharp outlines of objects, there is no color saturation. At the same time, the rather rare smoke on the left side of the image is no longer visible, but a denser, outlined haze in the center remains noticeable.

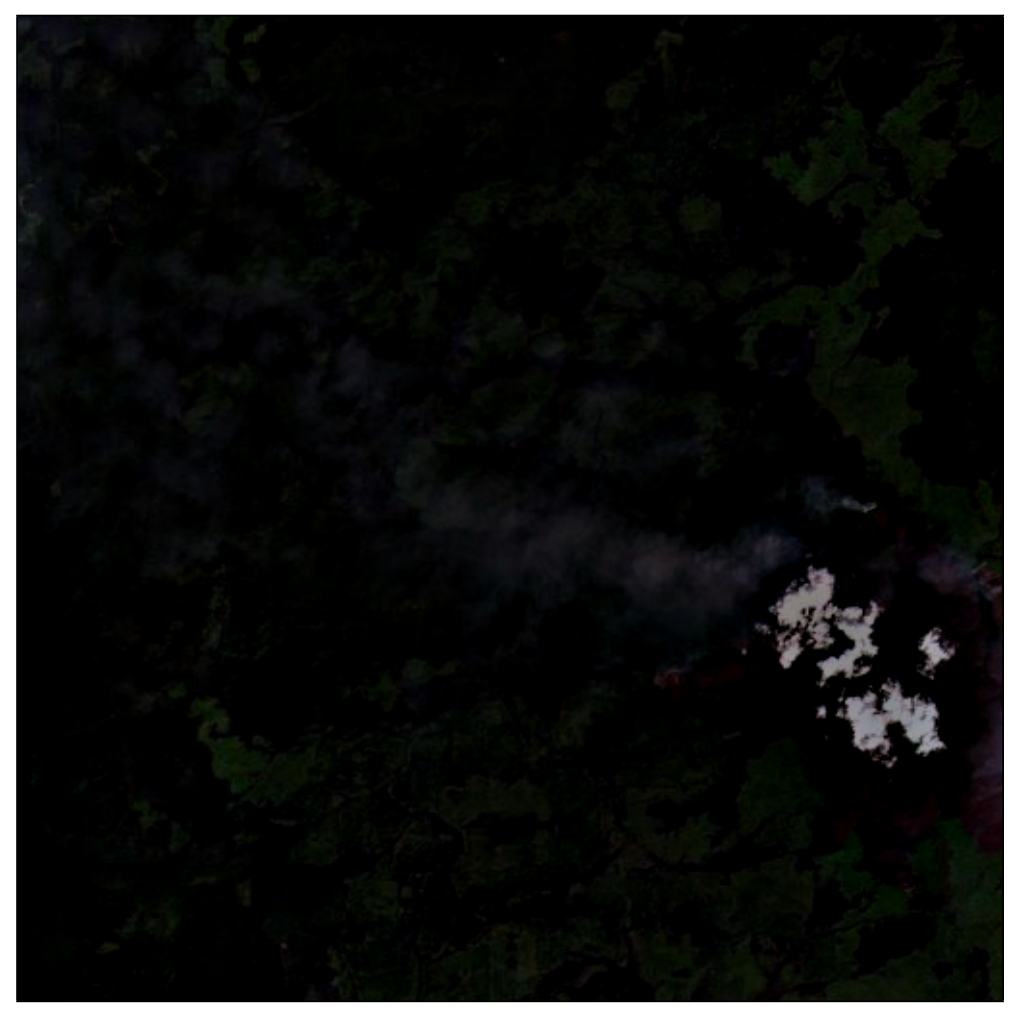

Fig. 3. The result of applying a Gaussian HPF with $D_{0}=4$ 
Now let's combine the obtained filtering result with the original image. The final result of digital processing is shown in Figure 4.

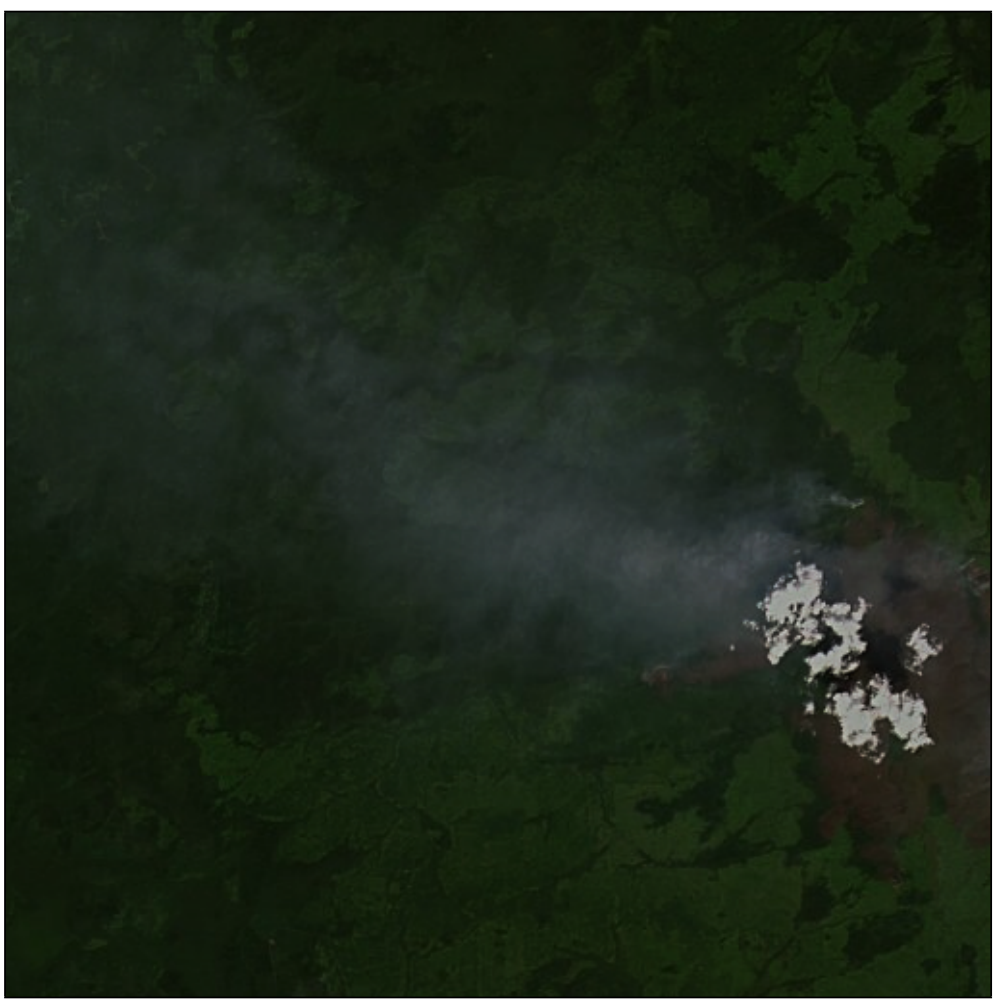

Fig. 4. Global high pass filtering result

\section{Conclusion}

On the example of a photo of a smoky surface of the Taseevsky district of the Krasnoyarsk Territory, a method of applying global high-frequency filtering to improve the image is shown. As a result of processing, the sparse haze on the left side of the original image becomes less noticeable. At the same time, dense smoke in the center became more rarefied, but did not disappear completely, since in the high-frequency spectrum the boundaries of this cloudiness have a significant effect on the image.

\section{Acknowledgments}

This work was carried out as part of the state assignment of the Ministry of Science and Higher Education of the Russian Federation (scientific code FSRZ-2020-0011).

\section{References}

1. E.A. Maltsev, E.E. Sirotin, D.A. Perfil'ev, G.M. Tsibul'skii, Journal of Siberian Federal University. Engineering \& Technologies 2, №5, 229 - 242 (2012) 
2. O.S. Tokareva, Processing and interpretation of Earth remote sensing data, 148 (2010)

3. R.C. Gonzalez and R.E. Woods. Digital Image Processing, (2012)

4. Snapshot of the Taseevsky district of the Krasnoyarsk Territory from 07.30.2020. Access mode: http://earthexplorer.usgs.gov/

5. M.V. Noskov, V.S. Tutatchikov, Pattern Recognition and Image Analysis, 25(1), 81-83 (2015)

6. M. Noskov, V. Tutatchikov, E3S Web of Conferences, 149, 02010 (2020) 